\title{
Propagation Impairments Modeling and QoS Parameters Characterization in a Ka-band Videoconferencing System
}

\author{
Reuben A. Farrugia, Student Member IEEE, Carl. J. Debono, Member, IEEE and \\ Paul Micallef, Member, IEEE
}

\begin{abstract}
Multimedia and Videoconferencing applications transmitted over satellite links are being pushed towards higher frequency bands where more usable bandwidth is present. The service availability of Ka-band satellite links is subject to considerable uncertainty, relying on channel phenomena such as interference and the unpredictability of local weather. This paper presents a model of the interference and the propagation impairments in Ka-band satellite communication systems. Moreover, a description of a simple method which may be used to derive quality of service $(\mathrm{QoS})$ parameters is given.
\end{abstract}

Keywords - Ka-band channel characterization, QoS, Propagation impairments, Multimedia communications.

\section{INTRODUCTION}

$\mathrm{I}_{\mathrm{a}}^{\mathrm{N}}$ the past decade, new and demanding satellite applications evolved leading to spectral congestion of the conventional frequency bands. Many Fixed Satellite Service (FSS) applications moved towards higher frequency bands which provide larger bandwidths at the cost of larger fades, noise and interference. These limitations make it difficult to provide Ka-band satellite services at availability and quality of service comparable to those at lower frequency bands [1].

The propagation impairments that effect Ka-band satellite links include cloud, gaseous, melting layer, rain and tropospheric scintillation [1]. Moreover, the effect of noise and cross-polar depolarization (for dual-polarized systems) should be considered for low fade-margin satellite systems. These impairments together with the carrier-to-noise ratio (CNR) were estimated statistically by means of the link budget, using propagation models and knowledge of the link characteristics.

A number of propagation models [1]-[13] were reviewed and those models which correlated most with

R. A. Farrugia is with the Department of Communications \& Computer Engineering at the University of Malta, Msida, MSD 06, Malta (e-mail: rrfarr@eng.um.edu.mt).

C. J. Debono is with the Department of Communications \& Computer Engineering at the University of Malta, Msida, MSD 06, Malta (phone: 356-23402076, fax: 356-21343577; e-mail: cjdebo@eng.um.edu.mt).

P. Micallef is with the Department of Communications \& Computer Engineering at the University of Malta, Msida, MSD 06, Malta (e-mail: pjmica@eng.um.edu.mt). experimental data were used in this work. The ITU-R P.840-3 [3] and ITU-R P.676-5 [4] recommendations, which model the attenuation due to clouds and atmospheric gases, have already been reviewed by COST Action 255 [14] and considered to be reliable models. The ITU-R P.618-7 [7] recommendation and Van de Kamp [10] model were used to derive the rain and tropospheric scintillation. These models were selected because when compared to experimental data they provided a close match. The melting layer was modeled using the model published by Zhang [5]. Its effect can be considered as negligible at low rain rates, however, its contribution may be important, especially for high availability systems.

There are few models which model the effect of crosspolar depolarization in the Ka-band. The ITU-R P.618-7 [7] recommendation proposed a simple model which models the effect of the combined effect of rain and ice depolarization. The effect of ice depolarization on its own was modeled according to the model proposed by Martellucci [13].

The paper is structured as follows: Section II provides information about the Ka-band system followed by simulations of different propagation impairment models, some of which were compared to available experimental data. The method used to characterize the QoS parameters is described in the following section. Finally, the results obtained are summarized in section IV.

\section{PROPAGATION IMPAIRMENTS}

Eutelsat's Hotbird 6 satellite system provides a number of Ka-band applications over the same coverage area. This geostationary (GEO) satellite was used to deliver point-topoint multimedia content and videoconferencing services between the University of Malta in Malta and its branch in the sister island of Gozo. It operates at an uplink frequency of $29.5 \mathrm{GHz}$ and a downlink frequency of 19.7 $\mathrm{GHz}$ utilizing dual linear polarization at an elevation angle of $48.71^{\circ}$.

The satellite coverage at both earth stations, illustrated in Figure 1, is approximately $5 \mathrm{~dB}$ less than its maximum. Thus, this system is more susceptible to signal degradation, and therefore proper prediction and characterization of this channel becomes of fundamental importance. In the following simulations a temperature of $18.26^{\circ} \mathrm{C}$ and a relative humidity of $64.71 \%$ were used. 


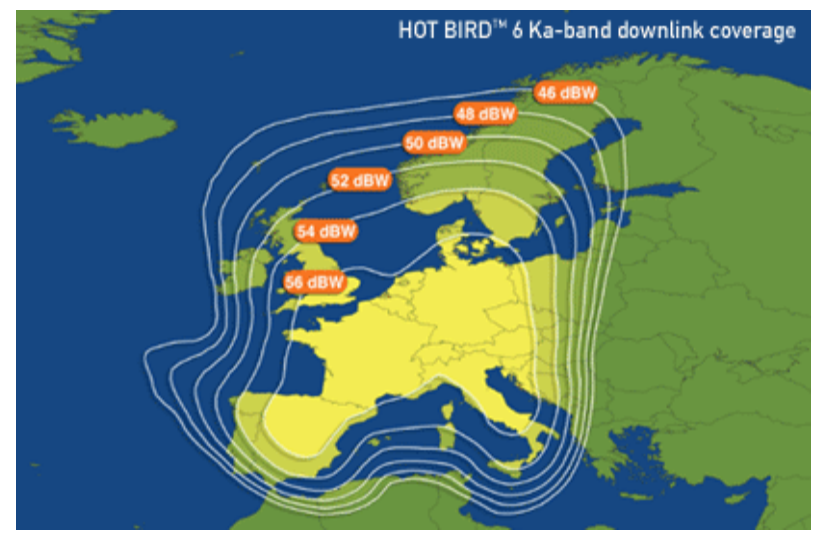

Fig. 1. The downlink coverage provided by Hotbird 6 .

\section{A. Gaseous Absorption}

Gaseous absorption, mainly due to oxygen and water vapor, is relatively small compared to other propagation phenomena. This phenomenon does not vary linearly with increase in frequency and may be significant at around $22.5 \mathrm{GHz}$, which is the resonant frequency of water vapor.

The ITU-R P.676-5 [4] model was used to predict the gaseous absorption. Figure 2 shows the predicted distribution of this system for both uplink and downlink.

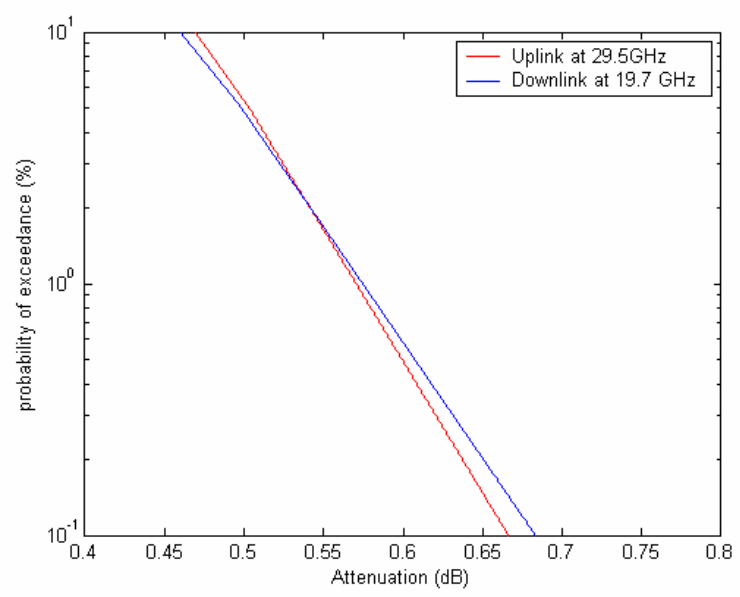

Fig. 2. Predicted gaseous attenuation in Malta.

\section{B. Clouds Attenuation}

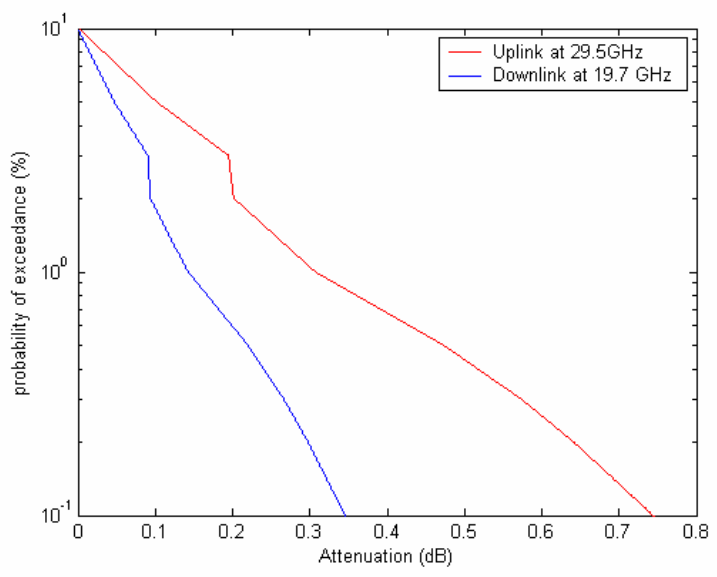

Fig. 3. Predicted cloud attenuation in Malta.

Clouds are composed of both water droplets and ice. Ice clouds do not significantly contribute to attenuation, while water droplets provide attenuation which is a function of frequency and water content. Figure 3 depicts attenuation values due to clouds exceeded for a certain range of probabilities in Malta according to ITU-R P.840-3 [3].

\section{Rain Attenuation}

Attenuation due to rain is the dominant propagation impairment at the Ka-band. Several models have been developed in the past, which yield results that agree well with experimental observations. The experimental data collected during the ACTS [14] project was used to identify the validity of the ITU-R P. 617-7 [7] model. Figure 4 illustrates the distribution of the predicted and measured rain attenuation in Norman, Oklahoma.

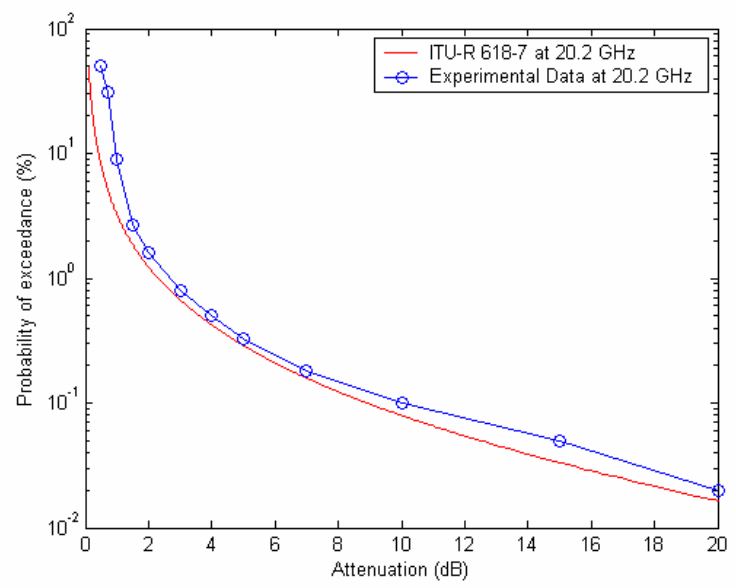

Fig. 4. Experimental and modeled rain attenuation a satellite link operating in Norman (OK).

Since the correlation is quite evident, it was used to model the expected attenuation due to rain in Malta, resulting in the distribution shown in Figure 5.

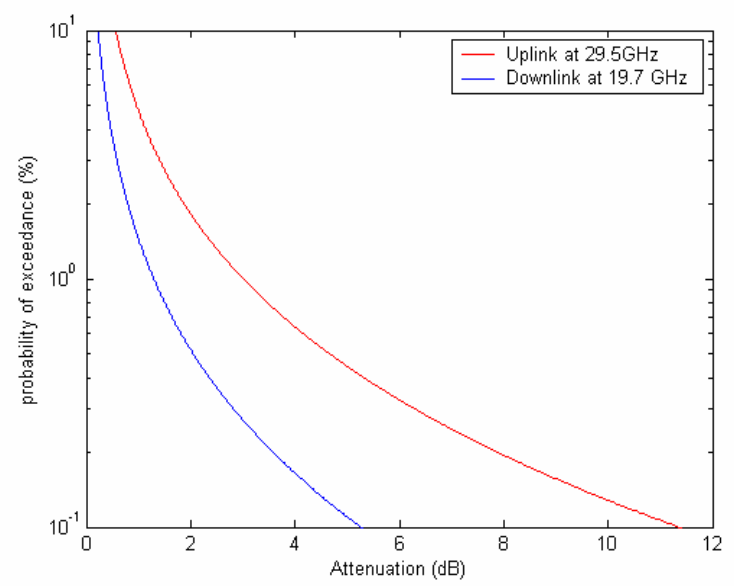

Fig. 5. Predicted rain attenuation in Malta.

\section{Melting Layer Attenuation}

The Melting Layer is a region of the atmosphere just below the $0^{\circ}$ isothermal level, where ice and snow turn into water drops. The Melting Layer attenuation is a function of rain rate and was modeled by W. Zhang [5] by approximating the Mie theory as a Power Law. The ITU-R P. 837-4 [16] rain rate model was integrated in this model to make the Zhang model a function of probability of exceedance. Figure 6 illustrates the expected attenuation due to Melting Layer in Malta. 


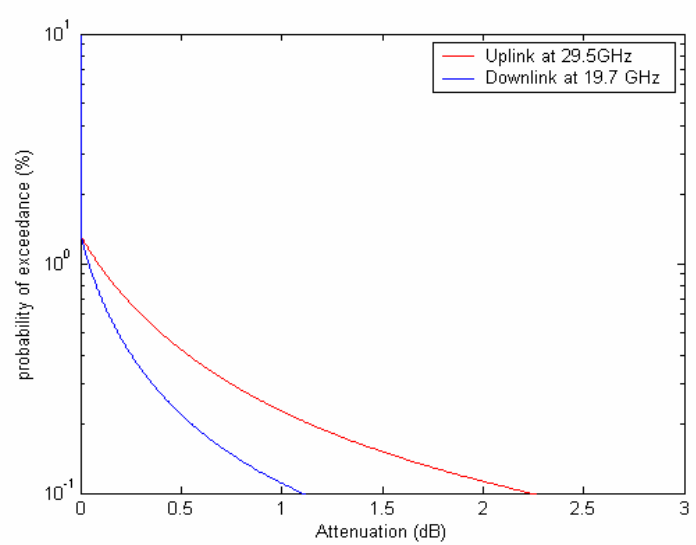

Fig. 6. Predicted melting layer attenuation in Malta.

\section{E. Tropospheric Scintillation}

Variations in the magnitude and profile of the refractive index of the troposphere lead to scintillation. This effect becomes significant at frequencies above $10 \mathrm{GHz}$ and at low elevation angles. The experimental data measured in Louvain-la-Neuve (Belgium), was used to correlate the Van de Kamp [10], [11] model. Figure 7 depicts the expected and measured data in Belgium.

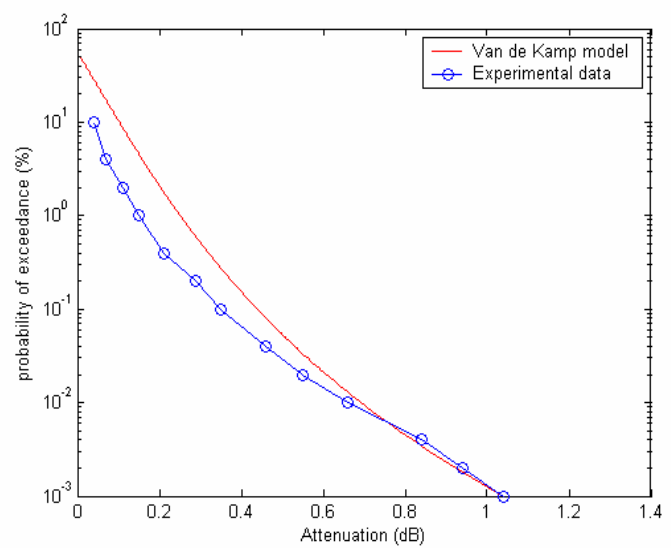

Fig. 7. The expected and measured scintillation fade in Louvain-la-Neuve [11].

The distribution of this model correlated better than other models and was therefore used to model the expected tropospheric distribution in Malta, as shown in Figure 8.

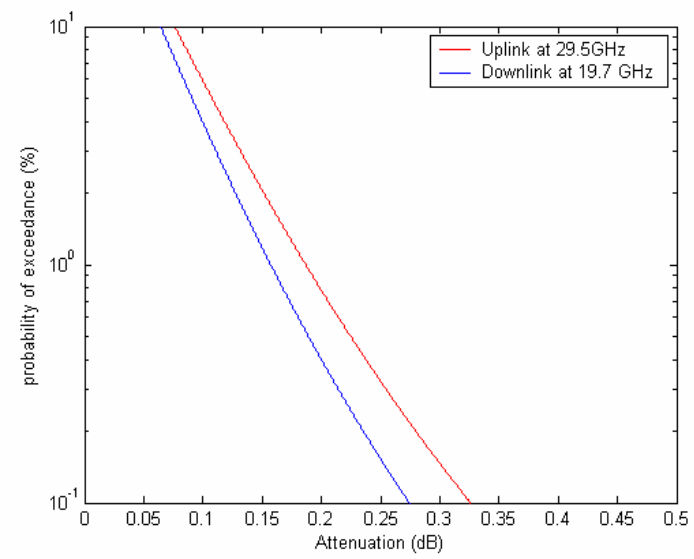

Fig. 8. Predicted tropospheric scintillation attenuation in Malta.

\section{F. Cross-Polar Depolarization}

Frequency reuse with orthogonal polarizations is often employed in modern satellites to double the capacity. However, cross-polar interference occurs in such systems, mainly because of the finite cross polarization discriminator (XPD) of the satellite and earth station antennas. Moreover, the dynamic depolarization effect of propagation mediums such as rain and ice further degrades the XPD of the satellite channel.

Raindrops falling take an oblate shape when precipitating through the atmosphere. As a result of this shape, different attenuation and phase are experienced by the orthogonal signal. Moreover, ice crystals present in the clouds further reduce the carrier-to-interference ratio. Figure 9 illustrates the predicted dynamic XPD in Malta when circular polarization is used.

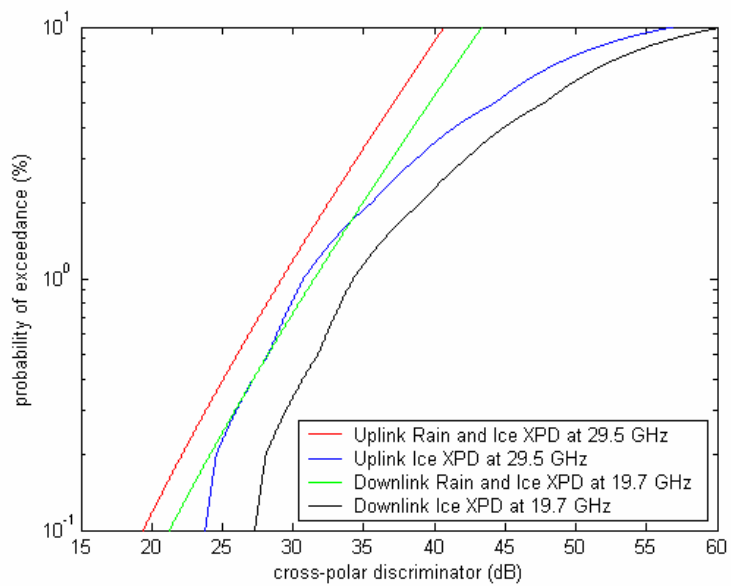

Fig. 9. Prediction of the ice and rain XPD in Malta.

\section{QOS CHARACTERIZATION}

The videoconferencing system used was made up of the videoconferencing equipment, a hub, a digital modulator and a computer on each site, as illustrated in the figure below.

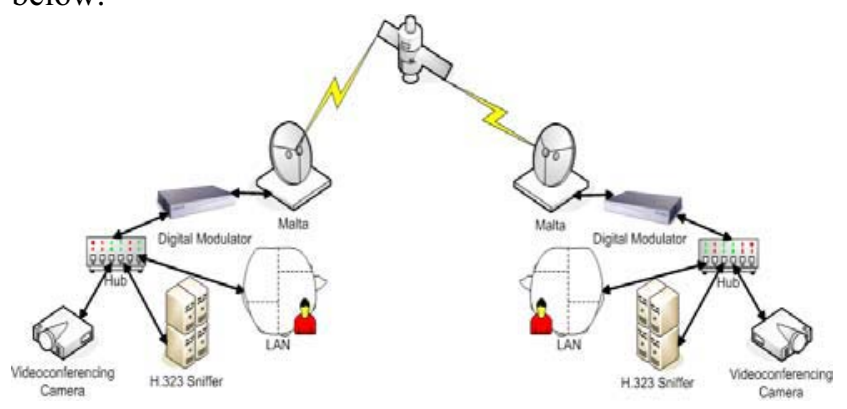

Fig.10. Videoconferencing System Architecture.

Both computers run as intrusion detection software called Snort [18] during the videoconferencing session. This application was configured to capture all the traffic which passes through the hub, including the videoconferencing H.323 packets transmitted between the two sites. Thus, two copies of the transmitted packet are logged by the system: the transmitted packet is logged on the transmitting site while the received packet is logged on the receiving site. 
Data logging should be captured with care as applying filters and logging data as text files slows down the Snort application. On high speed networks congestion may occur, resulting in packets being dropped by the network card of the computer.

To avoid packets being dropped, no filters were applied to Snort. Moreover, the captured packets are logged in TCPDUMP format to a single binary file. These files include the time stamp of each individual packet, accompanied by the Physical layer frame. Once the videoconferencing session is complete, the binary files are uploaded to a server, using the FTP protocol, where the data is processed offline.

After the collected data is filtered from the unwanted data, the transmitted and received packets are synchronized. These packets are compared and the required QoS parameters are calculated.

\section{Results}

The method described above was used to capture a live videoconferencing session which lasted about 30 minutes. This data was collected under clear sky conditions with the following meteorological data: Temperature $17^{\circ} \mathrm{C}$, Relative Humidity $73.0 \%$ and Atmospheric Pressure $1018 \mathrm{hPa}$.

TABLE 1: QOS PARAMETERS

\begin{tabular}{l|r}
\hline Parameter & Value \\
\hline Total number of bytes & $173.66 \mathrm{Mbytes}$ \\
Latency & $350.7 \mathrm{~ms}$ \\
Throughput & $786.96 \mathrm{kbps}$ \\
Packets lost & 0 \\
Bit Error Rate (BER) & 0 \\
\hline
\end{tabular}

A total of $1.389 \times 10^{9}$ bits were collected during the videoconferencing session, and the QoS parameters are summarized in Table 1. No bits in error were observed in this channel. This is because data transmission via satellite is very robust offering a maximum BER in the range of $10^{-8}-10^{-11}$ [17], [19]. However, as illustrated in the figure below, the CNR degradation is quite significant under precipitation, and this will degrade both the BER and Frame Error Rate (FER).

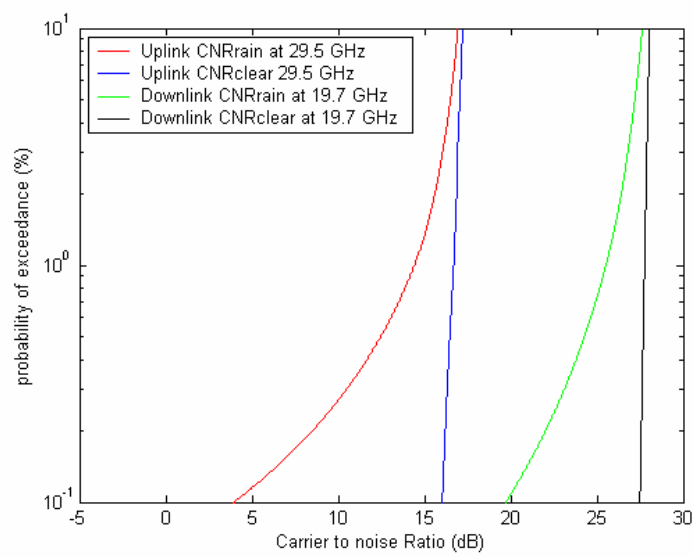

Fig. 11. Predicted Carrier-to-Noise Ratio in Malta when considering thermal noise and cross-polar depolarization as interfering sources.

\section{CONCLUSION}

Ka-band channel propagation modeling has been discussed in relation to satellite communication systems operation in Malta. More data should be collected using the above mentioned method, especially during precipitation to further enhance and validate the developed model. In the near future, the QoS parameters will also be included in the model and a study of their variation with channel usage and network configuration will be pursued.

\section{ACKNOWLEDGMENT}

The project was funded by the EU $6^{\text {th }}$ Framework Programme (FP6) project TWISTER. The authors would like to thank Dr. Martellucci for the provision of data required for the ice depolarization model.

\section{REFERENCES}

[1] A. D. Panagopoulos, P. M. Arapoglou and P. G. Cottis, "Satellite Communications at $\mathrm{Ku}, \mathrm{Ka}$, and $\mathrm{V}$ bands: Propagation Impairments and Mitigation Techniques", IEEE Communications Surveys \& Tutorials: Third Quarter 2004, Available: www.comsoc.org/pubs/surveys.

[2] E. Salonen and S. Uppola, "New Prediction Method of Cloud Attenuation”, Electronic Letters, vol. 27, pp.1106-08, Jun. 1991.

[3] ITU Recommendation ITU-R P.840-3, "Attenuation due to Cloud and Fog", 1999

[4] ITU Recommendation ITU-R P.676-5, "Attenuation by Atmospheric Gases", 2001

[5] W. Zhang, S. I. Karhu and E. T. Salonen, "Prediction of Radiowave attenuation due to melting layer of precipitation", IEEE Trans. Antennas and Propagation, vol. 42, pp. 492-500, Apr. 1994

[6] R. K. Crane, "Prediction of Attenuation by Rain", IEEE Trans. Communications, vol. COM 28, pp. 1717-1733, Sept. 1980.

[7] ITU Recommendation ITU-R P. 618-7, "Propagation data and prediction methods required for design Earth-space telecommunication systems", 2001

[8] A. Dissanayake, J. Allnutt and F. Haidara, "A Prediction model that combines rain attenuation and other propagation impairments along Earth-satellite paths", IEEE Trans. Antennas and Propagation, vol. 45, pp. 1546-57, Oct. 1997.

[9] I. E. Otung, "Prediction of Tropospheric Amplitude Scintillation on a Satellite link", IEEE Trans. Antennas and Propagation, vol. 44, pp. 1600-1608, Dec. 1996

[10] M. J. L. Van de Kamp, J. K. Tervonen, E. Salonen and J. P. Baptista, "Improved Models for Long-Term-Prediction of Tropospheric Scintillation on Slant Paths", vol. 47, pp. 249-60, Feb. 1999.

[11] M. J. L. Van de Kamp, "Climatic Radiowave Propagation Models for the design of Satellite Communication Systems", Ph.D. dissertation, Eindhoven University of Technology, Nov. 1999.

[12] Y. Karasawa, M. Yamada and J. Allnutt, "A New Prediction Method for Tropospheric Scintillation on Earth-Space Paths", IEEE Trans. Antennas and Propagation, vol. 36, pp. 1608-14, Nov. 1988.

[13] A. Martellucci, J. P. V. Poiares Baptista and G. Blarzino, "New climatological databases for Ice depolarization on satellite radio links", COST 280, $1^{\text {st }}$ International Workshop, Jul. 2002.

[14] COST Action 255, "Fixed Propagation Modelling", Final Report, Part 2, Mar. 2002

[15] R. Crane and A. Dissanayake, "ACTS Propagation Experiment: Attenuation Distribution Observations and Prediction Model Comparisons", Proceedings of the IEEE, vol. 85, pp. 875-892, Jun. 1997.

[16] ITU Recommendation ITU-R P.837-7, "Characteristics of precipitation for propagation modeling", 2003.

[17] COST Action 255, "Test Cases Involving Fixed Satellite Links", Final Report, Part 6, Mar. 2002

[18] SNORT is a network intrusion detection application software, Available: http://www.snort.org

[19] EUTELSAT Technical Guide Annex B-Overview of DVB, EUTELSAT, Paris. 1999. 